\section{ECG Spectrum Analysis Predicts Return of Spontaneous Circulation following Prolonged Ventricular Fibrillation}

\author{
Menegazzi J], Watanabe S, Klain M, Doctor A \\ Center for Emergency Medicine of Western Pennsylvania \\ Pittsburgh, Pennsylvania, USA \\ Division of Emergency Medicine, University of Pittsburgh \\ Pittsburgh, Pennsylvania, USA
}

Purpose: To determine the utility of electrocardiogram (ECG) spectrum analysis in predicting the return of spontaneous circulation (ROSC) in a swine model of out-of-hospital cardiac arrest.

Methods: This was a prospective experimental trial, conducted in an animal laboratory, using 10 mixed-breed domestic swine of either gender (weighing 19.0 to $23.7 \mathrm{kgs}$ ). Animals were sedated, intubated, anesthetized, and instrumented for monitoring of arterial and central venous pressures, and ECG. Ventricular fibrillation (VF) was induced using a bipolar pacing catheter, and was untreated for eight minutes. Following eight minutes of no-flow, advanced cardiac life support (ACLS) was begun. ECG was amplified with the HP Model $8811 \mathrm{D}$ ECG/HIS Conditioner with a bandpass filter of $0.5-30 \mathrm{hz}$. A $12-$ bit $\mathrm{A} / \mathrm{D}$ converter and Wave Master II visual wave editor were used to sample at a frequency of $500 \mathrm{~Hz}$. Spectrum analysis applied Fast Fourier Transformation with 2K bits of sampling points. Frequency and amplitude were measured at the maximum amplitude points.

Results: Five of six cases with a combined frequency $\geq 10 \mathrm{~Hz}$ and amplitude $\geq 20 \mathrm{mV}$ achieved ROSC. In $33 / 33$ trials with combinations of frequency and amplitude below these thresholds, all failed to achieve ROSC.

Conclusions: In this swine model of prolonged cardiac arrest, ECG spectrum analysis correctly predicted ROSC in 38/39 trials (positive predictive value $=83 \%$, negative predictive value $=$ $100 \%$ ). Validation of this model using human, automatic external defibrillator tapes is in progress. These findings indicate that real-time prediction of ROSC may be possible, and may be useful in guiding electrical and pharmacotherapy by EMS personnel.

\section{Performance of Police First-Responders Trained in the Use of an Automated External Defibrillator}

\author{
Davis E, Menegazzi J, Davis C \\ University of Pittsburgh \\ Pittsburgh, Pennsylvania, USA
}

Hypothesis: The purposes of this study were to evaluate the performance of first-responder police officers in the use of an automated external defibrillator (AED) after a short, standardized training program, and to compare their performance to established rates for EMT-AED use.

Methods: This is a one group, time series design from February 1992 to November 1992. The participants were 157 police officers from seven departments who serve a suburban population, routine!y respond to medical emergencies, and who previously were trained only in CPR and first-aid. Each officer underwent a 4.5-hour course on AED theory and use. All passed an initial and quarterly practical. Performance was evaluated by 1) written exam prior to training, post-training, and six months following completion of training; 2) assessment by on-scene ALS providers; and 3) review of AED audio and rhythm tape utilizing preset criteria. Data were analyzed with the one sample, exact binomial test, with 45 cases forming the group for analysis. An alpha value $<.05$ was considered significant.

Results: Average scores for the three written tests were $69.4 \%$, $93 \%$, and $88 \%$. Proportions for the study group's performance are presented with $95 \%$ confidence intervals, with comparative probability based on previously published EMT-AED rates.

$\begin{array}{lcclll}\text { Factor } & \begin{array}{c}\text { EMT AED } \\ (\%)\end{array} & \begin{array}{c}\text { Police AED } \\ \text { (\%) }\end{array} & \begin{array}{l}\text { 95\% } \\ \text { Cl }\end{array} & \text { p-value } \\ \text { Correct protocol } & & & & & \\ \text { sequence } & 93 / 273(34) & 39 / 45(85.4) & .73-.95 & .0000001 \\ \text { No error rate } & 95 / 273(35) & 28 / 45(62) & .47-.76 & .00018 \\ \text { CPR delay } & 96 / 273(35) & 12 / 45(27) & .15-.42 & .16 \\ \text { Failure to clear } & 63 / 273(9) & 4 / 45(8.9) & .03-.21 & .013 \\ \text { Failure to re-analyze } & 25 / 273(9) & 3 / 45(6.7) & .01-.18 & .41\end{array}$

Police errors most often involved airway $(14 \%)$ and re-analysis $(25.6 \%)$. In no case did an officer fail to follow an AED prompt.

Conclusion: Minimally trained police officers can perform AED as well as or better than EMTs. Police officers followed the correct protocol sequence and had no errors significantly more often than did EMTs. Future training strategies and product design may stress airway and prompt CPR. 